\title{
Research on the Effective Image Retrieval Algorithm based on the Edge Detection and Morphological Analysis
}

\author{
Xinxin Xie $^{1, a}$ and Wenzhun Huang ${ }^{1, b^{*}}$ \\ ${ }^{1}$ School of Information Engineering, Xijing University, Xi'an 710123, China \\ a346148500@qq.com, bhuangwenzhun@xijing.edu.cn \\ *The corresponding author
}

Keywords: Morphological analysis, Edge detection, Image retrieval, Effective methods

\begin{abstract}
In this paper, we conduct research on the effective image retrieval algorithm based on the edge detection and morphological analysis. In the process of image retrieval, the traditional method, because of the disturbance to the environment and the filming equipment considered inadequate, because the filming resolution, basic brightness, light, the influence of shooting angle and the filming equipment to the characteristics of its image as a whole diversification, unable to effectively retrieve images or inaccurate image retrieval problem. Obtaining image classifier, through the classifier for calculation, the relative distance between all the images according to their distance from big to small order, output the final result. Under the background, we integrate edge detection and morphological analysis to form the revised version of the issues that is meaningful.
\end{abstract}

\section{Introduction}

The rapid development of the computer technology making the image database application in a short period of time to become new research area as content-based image retrieval is a new kind of retrieval technology, using the image features such as color, texture and shape. In an image database based on content retrieval and the user can define the image characteristics of according to oneself, to find the similar or related images. People more and more demand for the image retrieval, and the traditional text-based retrieval technology is not suitable for large-scale image collection, image retrieval based on content index directly extracted from the image characteristics, feature extraction and indexing can automatically by computer that avoid subjectivity of the artificial description and reduce workload.

System A

Client Side
System B

Server Side

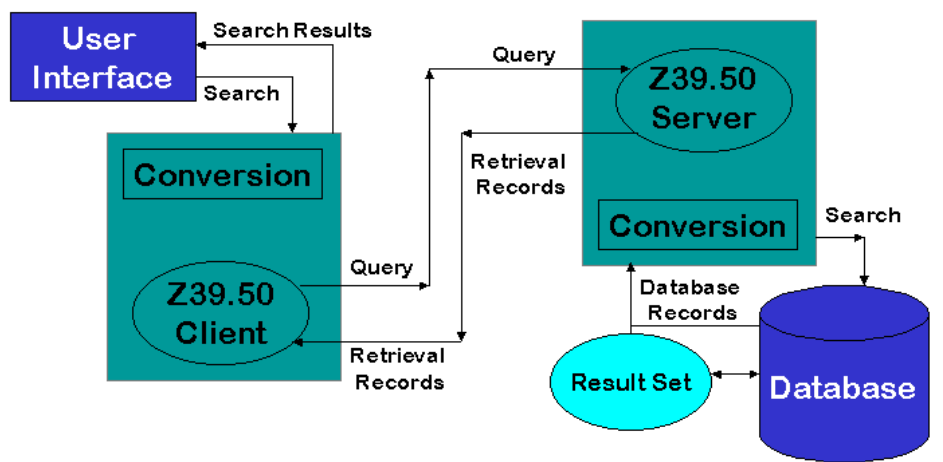

Figure 1. The Architecture of the Information Retrieval System

As demonstrated in the figure one, we illustrate the architecture of modern information retrieval system. Theoretically, the methodologies for retrieval of the information could be started from listed aspects. (1) Based on the retrieval of objects. Image retrieval based on the object of the current is still in research stage, the main research thought is based on the global characteristics of the image or the images contained object attributes for image retrieval. (2) Based on the texture characteristics of the retrieval. In image retrieval, object recognition and scene used the description of characteristics as the 
texture is one of the important characteristics. Texture was originally used to describe the concept of appearance of the plant fiber, in the image processing used to describe the regional spatial distribution characteristics of the pixel grayscale. (3) Based on color feature retrieval. In the process of retrieval, the characterization of basic color description usually has two kinds of methods: histogram and binary vector. Histogram is mainly describe the spatial distribution of the image color, binary vector mainly describe the characteristics of the image element. In the similarity comparison available Mahalanobis distance. (4) Based on the shape of the feature retrieval. Shape feature is one of the core features of the image and human visual system is one of key information of object recognition when the recognition, the shape of the image information changes over the image color is characteristic of stable objects.

In this article, we conduct research on the effective image retrieval algorithm based on the edge detection and morphological analysis. Mathematical morphology for image processing is based on the concept of placing the structure elements, the choice of structural elements and image of the close relations between some information of the different structural elements can complete different image analysis, and get a different result. For the optimization of morphological edge detection method is mainly used multiple structural elements of the different scale multiple morphological corrosion, that expansion calculation. In the sub-sections, we will discuss the issues in detail.

\section{Our Proposed Algorithm}

The Image Modeling. Image-based modeling technology is a hot research topic in the field of basic computer graphics and computer vision, it studies how to use a single image or video image sequence, the various clues information, restore the 3D model of the object or the scene. The technology in the digital preservation of cultural relics, film and television production, games, entertainment, industrial design, medical rehabilitation, and other fields has a very broad application prospects [1-2].

Modeling is the scene dynamic character, interactivity and external shape of the abstract; Describe the generation is to structure the model. Real-time graphics rendering technology in virtual scene is essentially a limited-time computing technology and the scene must be made within the given time. Complex scene modeling difficulty and the scene rendering speed slow is one of the difficulties in practical application of virtual reality. In order to improve the movement of the virtual scene model display speed, high-performance CPU and the graphics acceleration component is the basic hardware requirements, at the same time also need to elaborate design algorithm in software. Authenticity and operability is real-time, and the key technologies of virtual scene model are shown.

Multiple level of detail model is a widely used in virtual reality in detail control method, and this method can effectively improve the running speed of the system and basic interactive performance, therefore this study established a virtual scene model database, when used according to the observer to the virtual scene model in different observation distance from fine to coarse degree different model is adopted. The corresponding function for measurement could be expressed as follows [3-4].

$$
D=\left(d_{0}-d\right) / d_{0}
$$

On the same virtual scene model of different LOD model in the different scenarios of the smooth transition, this paper models the interpolation algorithm, the algorithm is simple in implementation and no special requirements for rough and fine model characteristics. Haven't found corresponding vertex in the fine model of each vertex, find the distance it recently on the rough model of vertices that establish the corresponding relationship between the two vertices as formula two.

$$
p=(1-t) p_{t}+t p_{n}
$$

To meet with lambert assuming the surface of the object, regardless of the light source direction, the surface of the same point in any direction to observe all have the same brightness, this is called the brightness uniformity. Therefore, by comparing the consistency of the object image brightness values under different angle of view, we can get the position of the 3D object surface point, to restore the 3D geometric model. Compared with other methods, the advantages of the method of brightness can be almost automatic get higher modeling precision. Because of the brightness information is the most 
abundant in the image, contains the largest amount of data information form. This enables brightness by calculation between different image matching and accurately calculates the depth of each point on the surface of object value. This makes it appear to be better able to geometric details on the surface of the object. In fact, for some meet the luminance method assumes that objects, existing methods can achieve close to 3D scanner modeling accuracy. And, compared with the general methods of shading, brightness illumination conditions no strict demand. But relative to the contour, zoom, interactive method and other methods, nor the image segmentation, change the focal length, such as basic manual interaction, which makes this method can meet the requirements of automatic.

The Edge Detection. The edge of image is one of the most basic and most important features of the images. Edge detection image of target and basic task is to find the real boundary line corresponding to the real edge and it has been a classic in the field of computer vision and image processing one of the research topics. Traditional classic algorithms are to extract the edge of the brightness gradient features alone, there will be discussed earlier to defect. In this paper, the edge detection method based on image characteristics, considering the image gradient information and phase information, with the brightness gradient and the direction of energy edge detection for image characteristics [5].

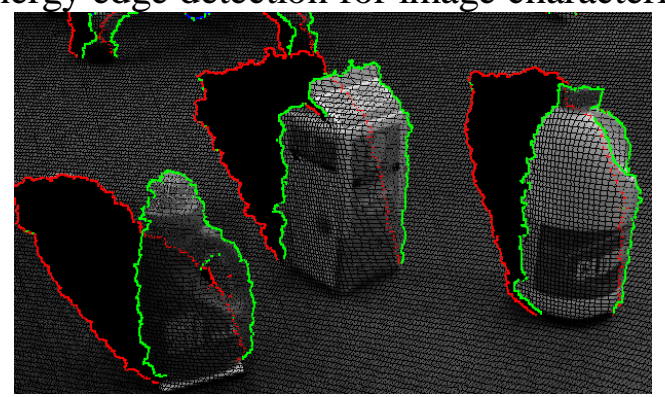

Figure 2. The Edge Detection Demonstration

Derivative operator have outstanding grayscale change effect, the image using derivative operator, the gray level change larger point calculate value is higher, so we will image the derivative operator operation value as the intensity of the corresponding boundary, so it can be set up by the derivative value threshold, extract the boundary point set that is demonstrated as formula 3.

$$
\left|\operatorname{grad}(f(x, y))=\left[\left(\frac{\partial f}{\partial x}\right)^{2}+\left(\frac{\partial f}{\partial y}\right)^{2}\right]\right|^{1 / 2}
$$

For simplification, we could denote the formula 4 as the following equation four.

$$
|\operatorname{grad}(f(x, y))|=\left|\nabla_{x} f\right|+\left|\nabla_{y} f\right|
$$

Considering the scale of the edge and direction, the extracted image features were as the function of the scale and the direction, so as to improve the flexibility and accuracy of detection. The brightness of the image gradient, histogram, torque, frequency spectrum, energy and other features can reflect the edge information, this article choose energy intensity gradient and direction for the basic edge feature extraction. Robert operator is one of the most simple operator as is a kind of using partial differential operator to find the edge of the basic operator, it uses the difference between the two adjacent pixels approximate diagonal direction gradient amplitude detection edge. Vertical edge detection effect is good in oblique edge with the high positioning accuracy, sensitive to noise [6].

Canny edge detection algorithm is by looking for local maximum values of the image gradient, the gradient is calculated using gauss function of first order differential. The essence of the algorithm is using a quasi-Gaussian function as smooth operation, and then to take the direction of the first order differential positioning derivative maximum. In the Canny method, respectively by two thresholds is strong and the weak edge detection edge; If and only if the weak edges and strong connection, the weak edge was output. Canny operator than Roberts operator, Sobel operator and Prewitt operator minimum algorithm of de-noising ability is stronger, to be able to achieve a better balance between noise and edge detection, can detect weak edges, but it is also easy to smooth out edge information. 
The Morphological Analysis. Mathematical morphology based on the morphological analysis of images, the basic idea is to use certain forms of structural elements to measure and extract the image of corresponding shape in order to achieve purpose of image analysis and recognition. Mathematical morphology image processing method has two basic operations: corrosion and inflation.

A collection based on the point of view is a natural result of morphology operator performance will be the main features, on the basis of geometric and traditional theory to resolve way to describe the performance of the basic operator. The explicit geometrical description of the characteristics of more suitable for the visual information processing and analysis, therefore, mathematical morphology is a direct relationship with the geometry-very attractive advantages. Using the mathematical morphology structure elements to measure and extract the image to be processed in the corresponding shapes and the corrosion and inflation are defined as the formula 5 and 6 , respectively.

$$
\begin{aligned}
& (I \oplus S E)(x, y)=\max \{I(x-k, y-l)+S E(k, l)\} \\
& (I \square S E)(x, y)=\min \{I(x+k, y+l)-S E(k, l)\}
\end{aligned}
$$

Through analysis on characteristics of artificial fuzzy image edge can be seen studied corrosion morphology filter operation can shrink away part of the image edge of "weak", keep on the edge of the "strong", for after a homomorphic filtering correction to enhance operation, the binary edge image of corrosion can shrink away homomorphic filtering is not normal region "weak" edge enhanced image, while retaining the homomorphic filtering to enhance the image tampering area "strong".

The Effective Image Retrieval Algorithm. System is the core image feature database as image features can be extracted from the image itself already also can be achieved through user interaction. The relationship between the user and the basic system works both ways: the user query request to the system, the system according to return query results query request, user through the relevant feedback to improve the query results query results that we should follow the listed trend. (1) Texture feature. Texture analysis methods in general can be divided into statistical method and structure. Statistical analysis based on the spatial distribution of intensity image color information. Structure analysis tries to find out the texture primitive, from primitive structure to explore the law of the texture. (2) Shape features. Shape is the most essential characteristics of depicting objects as is also one of the most difficult to describe characteristics, major difficult in interested in the image of target segmentation. Generally speaking, the shape contor features and regional features of two kinds of representation. (3) Color features. In addition to the color histogram, said the commonly used color features and color moment, color, color aggregation vector, color correlogram, etc.

Color is not like other information to reveal the nature of the object, but the color feature of image has its special characteristics, that in some cases the use of color image retrieval has a high efficiency and accuracy. If color feature extraction in a pair of images directly, very large amount of calculation, so we need to use as long as the color. As a result, the color quantization is the necessary operating statistical color features. Color quantization is the purpose of reduce the digital image in color, use as little as possible the color of the express image of color visual information. Commonly used with the interval of quantitative method and class clustering method as the follows [7-8].

$$
\begin{aligned}
& D_{\text {one }}=\left|f_{Q}-f_{I}\right|=\sum_{R, G, B}\left|\mu_{Q}-\mu_{I}\right| \\
& D_{\text {two }}=\sqrt{\left(f_{Q}-f_{I}\right)^{2}}=\sqrt{\sum_{R, G, B}\left(\mu_{Q}-\mu_{I}\right)^{2}}
\end{aligned}
$$

Content-based image retrieval can be divided into two basic steps: image feature extraction and matching. The use of color features for image retrieval is one of the keys to the extraction of color features, color characteristics of image can be all sorts of color distribution and the proportion of the color spatial distribution, etc. A pair of image color information, said by several main color is using the primary color with the least amount of color to represent the original image, using clustering algorithm can get the appropriate amount of the main color, not only reduced the feature dimension, but also can accurately express the original image, to achieve the balance of precision and dimension. 
The reference color histogram according to points of the color sorting, namely, in turn, find out the main color, main color, secondary color, and so on, if the same points according to its origin and color space distance to determine successively and the revised measurement standard is shown as below.

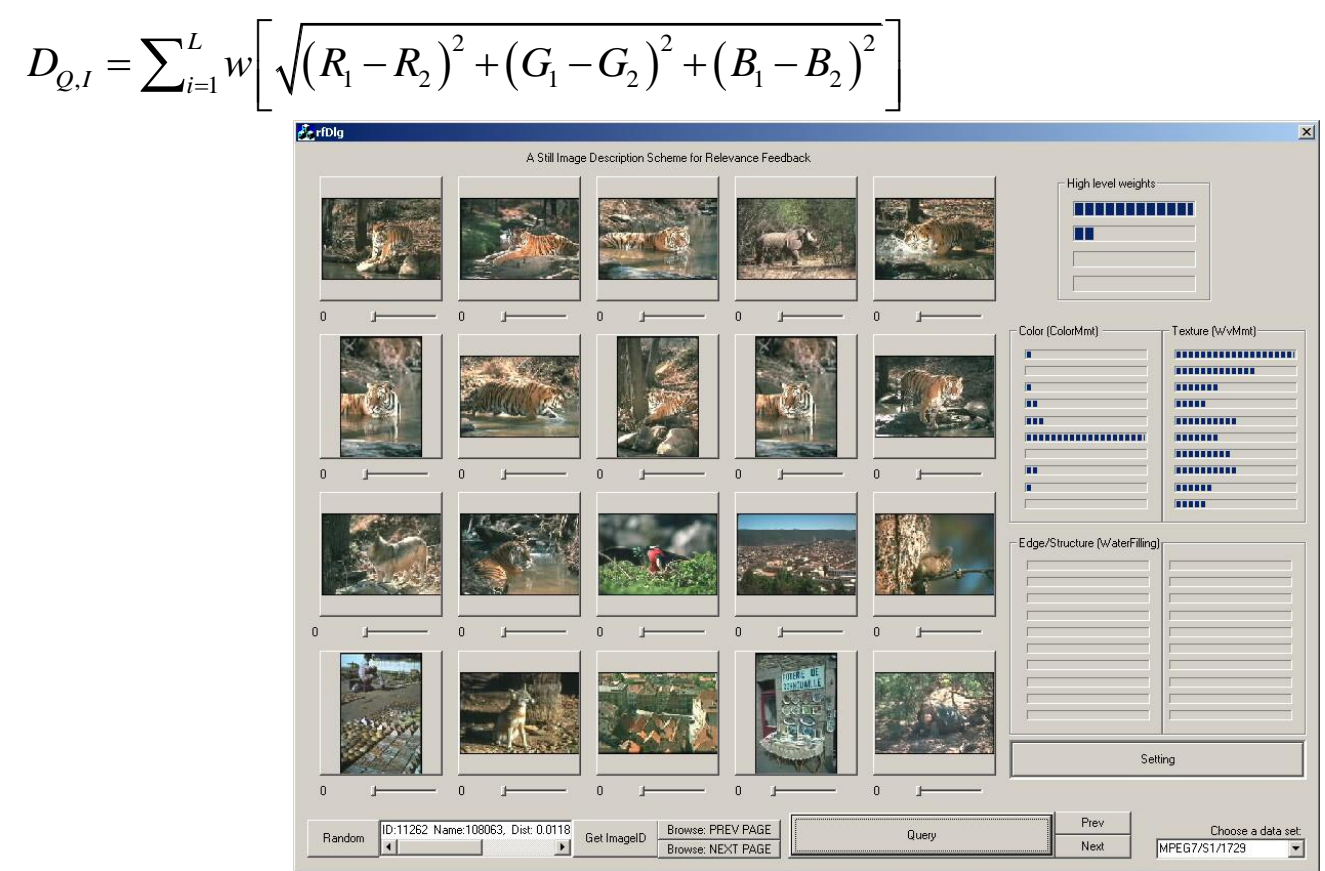

Figure 3. The Verification and Simulation of the Image Retrieval System

\section{Conclusion}

In this paper, we conduct research on the effective image retrieval algorithm based on the edge detection and morphological analysis. Content-based image retrieval technology is still in a primary exploration stage. Although according to image low-level features such as color, texture and shape retrieval had certain progress, but still limited to the specific applications, performance remains to be further improved. According to the characters of high level, such as time, place, and the integration of retrieval is still considerable difficulty. In the future research, we will conduct more in-depth analysis for the enhancement of the corresponding result.

\section{References}

[1] S. R. Dubey and A. S. Jalal: International Journal of Applied Pattern Recognition, Vol. 2 (2015) No. 2, p. 160.

[2] M. Xia, W. T. Lu, J. Yang, Y. Ma, W. Yao and Z. C. Zheng: Neurocomputing, Vol. 160 (2015) No. Jul.21, p. 238.

[3] M. M. Ji, Y. F. Feng, J. Xiao, Y. T. Zhuang, X. S. Yang and J. J. Zhang: Neurocomputing, Vol. 169 (2015) No. Dec. 2, p. 23.

[4] D. A. Hanauer, Q. Z. Mei, J. Law, R. Khanna and K. Zheng: Journal of biomedical informatics, Vol. 55 (2015), p. 290.

[5] Y. J. Xia, L. M. Zhang, X. L. Li and R. R. Ji: IEEE Transactions on Industrial Electronics, Vol. 62 (2015) No. 2, p.1301.

[6] M. Volkov, G. Rosman, D. Feldman, J. W. Fisher and D. Rus: IEEE International Conference on Robotics and Automation (Seattle, WA, USA, May 26-30, 2015), p.3638. 
[7] P. Kulkarni, J. Zepeda, F. Jurie, P. Perez and L. Chevallier: IEEE International Conference on Acoustics, Speech and Signal Processing (South Brisbane, Queensland, Australia, April 19-24, 2015), p. 1379.

[8] W. Guo, F. Tokihiro and S. Ninomiya: Plant methods, Vol. 11 (2015) No. 1, p. 1. 